\title{
Subthreshold $\Xi^{-}$Production in Collisions of $\mathrm{p}(3.5 \mathrm{GeV})+\mathrm{Nb}$
}

\author{
G. Agakishiev ${ }^{7}$, O. Arnold ${ }^{9}$, A. Balanda ${ }^{3}$, D. Belver ${ }^{18}$, A. V. Belyaev ${ }^{7}$, J. C. Berger-Chen ${ }^{9}$, A. Blanco ${ }^{2}$,
} M. Böhmer ${ }^{10}$, J. L. Boyard ${ }^{16}$, P. Cabanelas ${ }^{18, a}$, S. Chernenko ${ }^{7}$, A. Dybczak ${ }^{3}$, E. Epple ${ }^{9}$, L. Fabbietti ${ }^{9}$, O. V. Fateev ${ }^{7}$, P. Finocchiaro ${ }^{1}$, P. Fonte ${ }^{2, b}$, J. Friese ${ }^{10}$, I. Fröhlich ${ }^{8}$, T. Galatyuk ${ }^{5, c}$, J. A. Garzón ${ }^{18}$, R. Gernhäuser ${ }^{10}$, K. Göbel ${ }^{8}$, M. Golubeva ${ }^{13}$, D. González-Díaz ${ }^{5}$, F. Guber ${ }^{13}$, M. Gumberidze ${ }^{5,16}$, T. Heinz ${ }^{4}$, T. Hennino ${ }^{16}$, R. Holzmann ${ }^{4}$, A. Ierusalimov ${ }^{7}$, I. Iori ${ }^{12, d}$, A. Ivashkin ${ }^{13}$, M. Jurkovic ${ }^{10}$, B. Kämpfer ${ }^{6, e}$, T. Karavicheva ${ }^{13}$, I. Koenig ${ }^{4}$, W. Koenig ${ }^{4}$, B. W. Kolb ${ }^{4}$, G. Kornakov ${ }^{18}$, R. Kotte ${ }^{6}$, A. Krása ${ }^{17}$, F. Krizek ${ }^{17}$, R. Krücken ${ }^{10}$, H. Kuc ${ }^{3,16}$, W. Kühn ${ }^{11}$, A. Kugler ${ }^{17}$, A. Kurepin ${ }^{13}$, V. Ladygin ${ }^{7}$, R. Lalik ${ }^{9}$, S. Lang ${ }^{4}$, K. Lapidus ${ }^{9}$, A. Lebedev ${ }^{14}$, T. Liu ${ }^{16}$, L. Lopes ${ }^{2}$, M. Lorenz ${ }^{8, c}$, L. Maier ${ }^{10}$, A. Mangiarotti ${ }^{2}$, J. Markert ${ }^{8}$, V. Metag ${ }^{11}$, B. Michalska ${ }^{3}$, J. Michel ${ }^{8}$, C. Müntz ${ }^{7}$, L. Naumann ${ }^{6}$, Y. C. Pachmayer ${ }^{8}$, M. Palka ${ }^{3}$, Y. Parpottas ${ }^{15, f}$, V. Pechenov ${ }^{4}$, O. Pechenova ${ }^{8}$, J. Pietraszko ${ }^{4}$, W. Przygoda ${ }^{3}$, B. Ramstein ${ }^{16}$, A. Reshetin ${ }^{13}$, A. Rustamov ${ }^{8}$, A. Sadovsky ${ }^{13}$, P. Salabura ${ }^{3}$, A. Schmah ${ }^{9, g}$, E. Schwab ${ }^{4}$, J. Siebenson ${ }^{9}$, Yu. G. Sobolev ${ }^{17}$, S. Spataro ${ }^{11, h}$, B. Spruck ${ }^{11}$, H. Ströbele ${ }^{8}$, J. Stroth ${ }^{8,4}$, C. Sturm ${ }^{4}$, A. Tarantola ${ }^{8}$, K. Teilab ${ }^{8}$, P. Tlusty ${ }^{17}$, M. Traxler ${ }^{4}$, R. Trebacz ${ }^{3}$, H. Tsertos ${ }^{15}$, T. Vasiliev ${ }^{7}$, V. Wagner ${ }^{17}$, M. Weber ${ }^{10}$, C. Wendisch ${ }^{4}$, J. Wüstenfeld ${ }^{6}$, S. Yurevich ${ }^{4}$, Y. V. Zanevsky ${ }^{7}$

(HADES collaboration)

\author{
${ }^{1}$ Instituto Nazionale di Fisica Nucleare - Laboratori Nazionali del Sud, 95125 Catania, Italy \\ ${ }^{2}$ LIP-Laboratório de Instrumentação e Física Experimental de Partículas , 3004-516 Coimbra, Portugal \\ ${ }^{3}$ Smoluchowski Institute of Physics, Jagiellonian University of Cracow, 30-059 Kraków, Poland \\ ${ }^{4}$ GSI Helmholtzzentrum für Schwerionenforschung GmbH, 64291 Darmstadt, Germany \\ ${ }^{5}$ Technische Universität Darmstadt, 64289 Darmstadt, Germany \\ ${ }^{6}$ Institut für Strahlenphysik, Helmholtz-Zentrum Dresden-Rossendorf, 01328 Dresden, Germany \\ ${ }^{7}$ Joint Institute of Nuclear Research, 141980 Dubna, Russia \\ ${ }^{8}$ Institut für Kernphysik, Johann Wolfgang Goethe-Universität, 60438 Frankfurt, Germany \\ ${ }^{9}$ Excellence Cluster 'Origin and Structure of the Universe', 85748 Garching, Germany \\ ${ }^{10}$ Physik Department E12, Technische Universität München, 85748 Garching, Germany \\ ${ }^{11}$ II.Physikalisches Institut, Justus Liebig Universität Giessen, 35392 Giessen, Germany \\ 12 Istituto Nazionale di Fisica Nucleare, Sezione di Milano, 20133 Milano, Italy \\ ${ }^{13}$ Institute for Nuclear Research, Russian Academy of Science, 117312 Moscow, Russia \\ ${ }^{14}$ Institute of Theoretical and Experimental Physics, 117218 Moscow, Russia \\ ${ }^{15}$ Department of Physics, University of Cyprus, 1678 Nicosia, Cyprus \\ ${ }^{16}$ Institut de Physique Nucléaire (UMR 8608), CNRS/IN2P3 - Université Paris Sud, F-91406 Orsay Cedex, France \\ ${ }^{17}$ Nuclear Physics Institute, Academy of Sciences of Czech Republic, 25068 Rez, Czech Republic \\ ${ }^{18}$ LabCAF F. Física, Univ. de Santiago de Compostela, 15706 Santiago de Compostela, Spain \\ ${ }^{a}$ also at Nuclear Physics Center of University of Lisbon, 1649-013 Lisboa, Portugal \\ ${ }^{b}$ also at ISEC Coimbra, 3030-199 Coimbra, Portugal \\ ${ }^{c}$ also at ExtreMe Matter Institute EMMI, 64291 Darmstadt, Germany \\ ${ }^{d}$ also at Dipartimento di Fisica, Università di Milano, 20133 Milano, Italy \\ ${ }^{e}$ also at Technische Universität Dresden, 01062 Dresden, Germany \\ ${ }^{f}$ also at Frederick University, 1036 Nikosia, Cyprus \\ ${ }^{g}$ now at Lawrence Berkeley National Laboratory, Berkeley, USA \\ ${ }^{h}$ now at Dipartimento di Fisica Generale and INFN, Università di Torino, 10125 Torino, Italy
}

(Dated: September 25, 2018)

\begin{abstract}
Results on the production of the double-strange cascade hyperon $\Xi^{-}$are reported for collisions of $\mathrm{p}(3.5 \mathrm{GeV})+\mathrm{Nb}$, studied with the High Acceptance Di-Electron Spectrometer (HADES) at SIS18 at GSI Helmholtzzentrum for Heavy-Ion Research, Darmstadt. For the first time, subthreshold $\Xi^{-}$production is observed in proton-nucleus interactions. Assuming a $\Xi^{-}$phase-space distribution similar to that of $\Lambda$ hyperons, the production probability amounts to $P_{\Xi^{-}}=(2.0 \pm 0.4$ (stat) \pm 0.3 (norm) \pm 0.6 (syst) $) \times 10^{-4}$ resulting in a $\Xi^{-} /\left(\Lambda+\Sigma^{0}\right)$ ratio of $P_{\Xi^{-}} / P_{\Lambda+\Sigma^{0}}=(1.2 \pm 0.3$ (stat) \pm 0.4 (syst) $) \times 10^{-2}$. Available model predictions are significantly lower than the estimated $\Xi^{-}$yield.
\end{abstract}

PACS numbers: 25.75.Dw, 25.75.Gz

The double-strange $\Xi^{-}$baryon (also known as cascade particle) when produced in elementary nucleon-nucleon (NN) collisions must be co-produced with two kaons ensuring strangeness conservation, $\mathrm{NN} \rightarrow \mathrm{N} \Xi \mathrm{KK}$. In fixed-target experiments, this requires a minimum beam energy of $E_{t h r}=$ $3.74 \mathrm{GeV}\left(\sqrt{s_{t h r}}=3.25 \mathrm{GeV}\right)$. In heavy-ion and even in 
nucleon-nucleus collisions cooperative processes are possible allowing for the production below this threshold. Above threshold and in heavy-ion reactions, the $\Xi^{-}$hyperons were measured over about three orders of magnitude of the centreof-mass energy covered by the LHC $\left(\sqrt{s_{N N}}=2.76 \mathrm{TeV}\right.$ [1]), RHIC $\left(\sqrt{s_{N N}}=62.4,200 \mathrm{GeV}[2,3]\right), \operatorname{SPS}\left(\sqrt{s_{N N}}=\right.$ $8.9,17.3 \mathrm{GeV}$ [4], $\sqrt{s_{N N}}=6.4-17.3 \mathrm{GeV}$ ) [5]), and AGS $\left(\sqrt{s_{N N}}=3.84 \mathrm{GeV}\right.$ [6] $)$ accelerators. The yield of multi-strange particles produced in nucleon-nucleus $(\mathrm{p}+A)$ and nucleus-nucleus $(A+A)$ collisions below their production threshold in NN collisions, is expected to be sensitive to the equation of state $(\mathrm{EoS})$ of nuclear matter, similar to single-strange hadrons [7,-9]. In heavy-ion reactions, the necessary energy for the production of multi-strange hyperons can be accumulated via multiple collisions involving nucleons, produced particles and short-living resonances. The corresponding number of such collisions increases with the density within the reaction zone the maximum of which in turn depends on the stiffness of the EoS.

Predictions of sub-threshold cascade production at energies available with the heavy-ion synchrotron SIS18 at GSI, Darmstadt, were made within a relativistic transport model [10]. The cross sections of the strangeness exchange reactions $\overline{\mathrm{K} Y} \rightarrow \pi \Xi(\mathrm{Y}=\Lambda, \Sigma)$, which were thought to be essential for $\Xi$ creation below NN threshold, were taken from a coupled-channel approach based on a flavor SU(3)-invariant hadronic Lagrangian [11]. At that time, no subthreshold $\Xi^{-}$ production was observed; the first announcement came from the HADES collaboration studying, at SIS18, $\mathrm{Ar}+\mathrm{KCl}$ reactions at a beam kinetic energy of $1.76 A \mathrm{GeV}\left(\sqrt{s_{N N}}=\right.$ $2.61 \mathrm{GeV}[12])$. The deduced $\Xi^{-} / \Lambda$ ratio was found substantially larger than any model prediction available at that time. Shortly after, other strangeness-exchange reactions, e.g. the hyperon-hyperon scattering processes, YY $\rightarrow \Xi \mathrm{N}$, exhibiting quite high cross sections, were figured out to largely account for this discrepancy [13, 14], while the reaction $\overline{\mathrm{K} Y} \rightarrow \pi \Xi$ was found negligible [13]. Also, a very recent investigation [15] of deep-subthreshold $\Xi$ production in nuclear collisions with the UrQMD transport model [16, 17] making use of the YY cross sections provided in ref. [13] showed that the hyperon strangeness exchange is the dominant process contributing to the $\Xi$ yield. However, the model could not satisfactorily explain the $\Xi^{-} / \Lambda$ ratio measured by HADES. Presently, no experimental data exist on $\Xi$ production in $\mathrm{p}+A$ interactions near threshold. The lowest energy so far at which $\Xi^{-}$production has been observed in collisions of $\mathrm{p}+\mathrm{Be}$ and $\mathrm{p}+\mathrm{Pb}$ is the maximum SPS energy $\left(\sqrt{s_{N N}}=17.3 \mathrm{GeV}[18]\right)$.

It would be interesting to learn which processes contribute mainly to subthreshold $\Xi$ production in case of nucleonnucleus collisions, which are considered as a link between elementary $\mathrm{NN}$ and heavy-ion collisions. For instance, in $\mathrm{p}+A$ reactions at a beam kinetic energy of $3.5 \mathrm{GeV}$ (fixed target, $\sqrt{s_{N N}}=3.18 \mathrm{GeV}$ ), the scattering of two incoherently produced hyperons appears rather improbable. Also, at first sight, direct double-hyperon production seems to be impossible, since the threshold of $\sqrt{s_{t h r, \Lambda \Lambda}}=3.22 \mathrm{GeV}$ for the channel requiring the lowest energy effort, $\mathrm{pp} \rightarrow \Lambda \Lambda \mathrm{K}^{+} \mathrm{K}^{+}$, is only marginally lower than the $\Xi$ threshold. However, already the consideration of a rather modest Fermi motion of the nucleons within the nucleus, i.e. a counter-motion against the projectile with a momentum of about $50 \mathrm{MeV} / c$ (being well below the Fermi momentum), would lift the available energy above both thresholds. Another possibility to gain the necessary energy would be the scattering of the proton at an object acting more massively than a single nucleon in the nucleus. Thus, for $\mathrm{p}(3.5 \mathrm{GeV})+A$ collisions, a target object $\mathrm{X}$ as heavy as only 1.11 nucleon masses is sufficient to reach the threshold energy of the final state $\mathrm{X} \Xi \mathrm{KK}$. Hence, the co-operation of two, or more, correlated target nucleons, e.g. bound in $\alpha$ particles, would allow for this kinematic effect. Correspondingly, a high collectivity of the target nucleus was already observed in deep-subthreshold kaon production in $\mathrm{p}+A(A=\mathrm{C}, \mathrm{Cu}, \mathrm{Au})$ collisions at a beam kinetic energy of $1.0 \mathrm{GeV}$ studied by ANKE at COSY-Jülich [19]. Also, in electron scattering experiments, ${ }^{12} \mathrm{C}(\mathrm{e}, \mathrm{e}$ 'p) at $4.627 \mathrm{GeV}$, performed at JLab [20], a surprisingly high fraction of nucleons, i.e. $20 \%$, are found to be strongly correlated, predominantly in the form of proton-neutron pairs. Finally, $\Xi$ production in $\mathrm{p}+A$ (and $A+A$ ) might happen via an exotic channel as $\eta \Lambda \rightarrow \Xi \mathrm{K}$, probably via the excitation and decay of a massive resonance as, e.g., $\Lambda^{*}(2000,2100, \ldots)$ [21]. Another possible scenario is to produce a non-strange heavy resonance with a mass sufficiently high to decay into $\Xi K K$. This hypothesis is motivated by the fact that in $\mathrm{p}+\mathrm{p}$ collisions at $3.5 \mathrm{GeV}$ beam kinetic energy a substantial fraction of the exclusive production of $\Sigma(1385)^{+}+\mathrm{K}^{+}+\mathrm{n}$ was found to proceed via an intermediate broad $\Delta^{++}$excitation at about $2000 \mathrm{MeV}$ [22].

In this Letter, we report on the first observation of subthreshold $\Xi^{-}$production in nucleon-nucleus collisions at $\sqrt{s_{N N}}-\sqrt{s_{t h r}}=-70 \mathrm{MeV}$. The experiment was performed with the High Acceptance Di-Electron Spectrometer (HADES) at the Schwerionensynchrotron SIS18 at GSI, Darmstadt. HADES, although primarily optimized to measure di-electrons [23], offers also excellent hadron identification capabilities [24-26]. A detailed description of the spectrometer is presented in ref. [27]. The present results are based on a dataset which was previously analyzed with respect to $\mathrm{e}^{+} \mathrm{e}^{-}$ [28] as well as to pion and $\eta$ [29], $\mathrm{K}^{0}$ [30] and $\Lambda$ [31] production in collisions of $\mathrm{p}+\mathrm{Nb}$ at $3.5 \mathrm{GeV}$. The main features of the apparatus relevant for the present analysis are summarized in ref. [31].

In the present experiment, a proton beam of about $2 \times 10^{6}$ particles per second with kinetic energy of $3.5 \mathrm{GeV}$ was incident on a 12 -fold segmented target of natural niobium $\left({ }^{93} \mathrm{Nb}\right)$. The data readout was started by different trigger decisions [29]. For the present analysis, we employ only the data of the first-level (LVL1) trigger, requiring a charged-particle multiplicity $\geq 3$ in the time-of-flight wall composed of plastic scintillation detectors. We processed about $N_{\mathrm{LVL} 1}=3.2 \times 10^{9}$ of such LVL1 events.

It is important to mention that $\Sigma^{0}$ hyperons decay almost exclusively into $\Lambda$ 's via the decay $\Sigma^{0} \rightarrow \Lambda \gamma$ (branching ratio 
$\left.B R=100 \%, c \tau=2.22 \times 10^{-11} \mathrm{~m} \mathrm{[21]}\right)$, with the photon not being detected in the present experiment. Hence, throughout the paper, any " $\Lambda$ yield" has to be understood as that of $\Lambda+\Sigma^{0}$. Correspondingly, in case of simulations, where the individual particle species are known, the yields of $\Lambda$ and $\Sigma^{0}$ hyperons are summed up.

In the present analysis, we identify the $\Xi^{-}$and $\Lambda$ hyperons through their weak decays $\Xi^{-} \rightarrow \Lambda \pi^{-}(B R=99.9 \%, c \tau=$ $4.91 \mathrm{~cm})$ and $\Lambda \rightarrow \mathrm{p} \pi^{-}(B R=63.9 \%, c \tau=7.89 \mathrm{~cm})$ [21], with the charged hadrons detected in HADES [12, 25, 31]. The long lifetimes cause a sizeable fraction of these particles to decay away from the primary vertex. The precision of the track reconstruction with HADES is sufficient to resolve these secondary vertices [12]. To allow for $\Lambda$ selection various topological cuts on single-particle and two-particle quantities were applied. These are i) a minimum value of the proton track ${ }^{1}$ distance to the primary vertex (p-VecToPrimVer), ii) the same for the $\pi^{-}\left(\pi_{1}\right.$-VecToPrimVer), iii) an upper limit of the $\mathrm{p}-\pi^{-}$ minimum track distance (p- $\pi_{1}$-MinVecDist), and iv) a minimum value of the $\Lambda$ decay vertex distance to the primary vertex ( $\Lambda$-VerToPrimVer). Here, the off-vertex cut iv) is the main condition responsible for the extraction of a $\Lambda$ signal. Starting with the moderate conditions as used in the previous high-statistics analysis of the $\Lambda$ phase-space distribution and polarization [31], a clear $\Lambda$ signal could be separated from the combinatorial background in the $\mathrm{p}-\pi^{-}$invariant-mass distribution. While in that analysis a signal-to-background ratio in the order of unity was sufficient, for the present $\Xi^{-}$ search we start with a higher $\Lambda$ purity ( $>85 \%$, cp. [12]). Hence, with the stronger cuts and the requirement of an additional $\pi^{-}$meson, the number of reconstructed $\Lambda$ hyperons decreases from about 1.1 million to 300,000. (No event containing clearly more than one $\Lambda$ was found.) Taking this still high-statistics $\Lambda$ sample, we started the $\Xi^{-}$investigation by combining - for each event containing a $\Lambda$ candidate (selected by a $\pm 2 \sigma$ window around the $\Lambda$ peak) - the $\Lambda$ with those $\pi^{-}$ mesons not already contributing to the $\Lambda$. The result was a structureless $\Lambda-\pi^{-}$invariant mass distribution. Hence, additional conditions were necessary: v) a lower limit on the 2nd $\pi^{-}$(potential $\Xi^{-}$daughter) track distance to the primary vertex $\left(\pi_{2}\right.$-VecToPrimVer), vi) an upper limit of the distance of the $\Xi^{-}$pointing vector w.r.t. the primary vertex ( $\Xi$-VecToPrimVer), vii) a maximum value of the minimum track distance of the $\Lambda$ and the 2 nd $\pi^{-}\left(\pi_{2}-\Lambda\right.$-MinVecDist), and viii) a minimum value of the distance of the $\Xi^{-}$vertex relative to the primary one ( $\Xi$-VerToPrimVer).

Starting with the cut settings used in our previous analysis of deep-subthreshold $\Xi^{-}$production in collisions of $\mathrm{Ar}+\mathrm{KCl}$ at $1.76 \mathrm{~A} \mathrm{GeV} \mathrm{[12]} \mathrm{and} \mathrm{optimizing} \mathrm{further} \mathrm{for} \mathrm{the} \mathrm{present}$ experiment which exhibits different multiplicities and phasespace distributions of the involved particles, we find a signifi-

\footnotetext{
${ }^{1}$ With "track" we mean the trajectory of a particle track extrapolated up to the relevant vertex.
}

cant narrow peak structure in the $\Lambda-\pi^{-}$invariant-mass distribution displayed in Fig. 11. (For convenience, we use identical mass and energy units.) The position is slightly lower by about $4 \mathrm{MeV}$ than the PDG value of $1321.71 \mathrm{MeV}$ [21], very probably due to a minor systematics uncertainty of the momentum calibration for charged particles in the inhomogeneous field of the toroidal magnet which leads to a slight $(<0.4 \%)$ phasespace dependence of the mass of the reconstructed weakly decaying mother particle as observed already for $\mathrm{K}^{0}$ and $\Lambda$ reconstruction [30, 31]. The width of the present peak, however, is well in agreement with the results of GEANT [32] simulations of about 2-3 MeV for $\Lambda$ and $\Xi^{-}$hyperons. More importantly, also the cut dependences of the yield of the potential $\Xi^{-}$match well those found from GEANT simulations (see below). Thus, we attribute the signal to the decay of the $\Xi^{-}$baryon.

The full curve in Fig. 11 shows the result of a fit to the data with a model function consisting of a Gaussian function for the peak and a polynomial function of 2 nd order for the combinatorial background (bg). Integration around the peak maximum within a window of $\pm 5 \mathrm{MeV}(\approx \pm 2 \sigma$, with $\sigma$ being the Gaussian width) we find $N_{\Xi^{-}}=90 \pm 18$ with the statistical error given. The signal-to-background ratio and the significance, signal $/ \sqrt{\text { signal }+ \text { bg }}$, amount to 0.39 and 5.0, respectively. Note that the raw $\Xi^{-}$yield per LVL1 event of $N_{\Xi^{-}} / N_{\mathrm{LVL} 1}=2.8 \times 10^{-8}$ is yet a factor seven smaller than the corresponding yield in $\mathrm{Ar}+\mathrm{KCl}$ reactions at $1.76 \mathrm{~A} \mathrm{GeV}$ [12]. We studied also the raw phase-space distribution of the

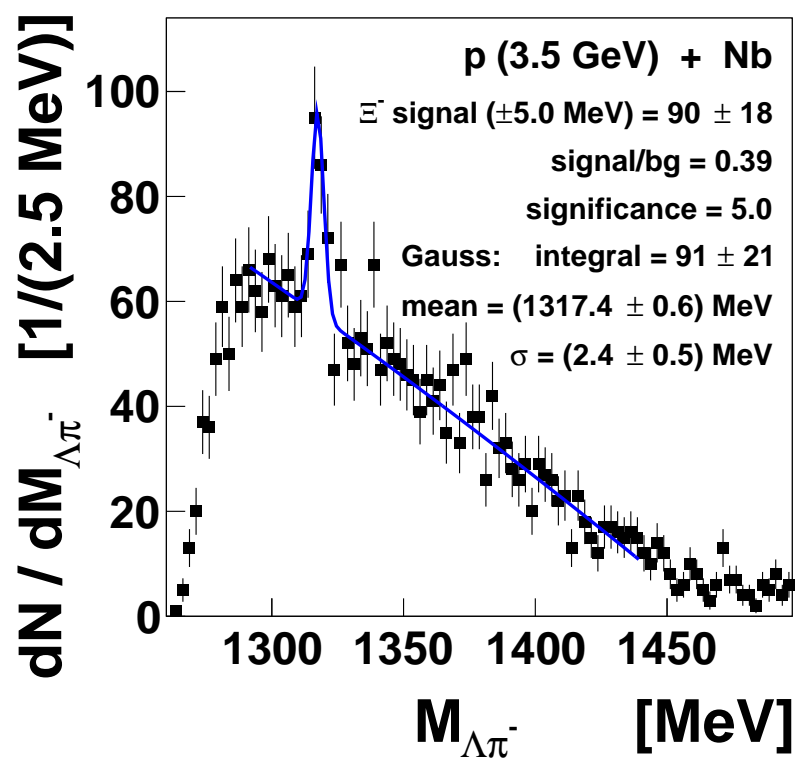

FIG. 1: The experimental $\Lambda-\pi^{-}$invariant-mass distribution. The error bars show the statistical errors. The curve represents a combination of a Gaussian and a polynomial function used to fit the data.

$\Xi^{-}$baryons. To that purpose, the yield within a window of 
$\pm 5 \mathrm{MeV}$ around the $\Xi^{-}$peak in Fig. 1 was selected, and the combinatorial background below the peak was subtracted with the help of a corresponding sideband analysis. The resulting transverse-momentum vs. rapidity distribution was found strongly biased by the HADES acceptance, i.e. essentially by the lower and upper polar angle limits of 18 and 85 degrees [29, 31]. This finding is confirmed by studies of the detector acceptance of simulated data and found to be rather independent of the input phase-space distributions. The mean value and the r.m.s. width of the experimental rapidity distribution amount to 0.54 and 0.16 , respectively. The corresponding values of the transverse-momentum distribution are $0.52 \mathrm{GeV} / c$ and $0.17 \mathrm{GeV} / c$, respectively.

Corrections for the finite acceptance and reconstruction efficiency were deduced from simulations. Thermo-statistically distributed $\Xi^{-}$baryons, characterized by a temperature parameter $T$, were generated with the event generator Pluto [33]. Since the phase-space distribution of the $\Xi^{-}$is not known, the experimental $\Lambda$ phase-space distribution (found to be strongly influenced by hyperon-nucleon collisions [31]) served as benchmark for the $\Xi^{-}$hyperon. Consequently, in Pluto we allowed for longitudinally shifted and elongated $\Xi^{-}$ phase-space distributions. For this purpose, two longitudinal shape parameter, i.e. the mean, $\langle y\rangle=0.3$, and the width, $\sigma_{y}=0.57$, following from a Gaussian fit to the $\Lambda$ rapidity distribution [31], are introduced. We investigated the $\Xi^{-}$ geometrical acceptance for a broad range of transverse and longitudinal shape parameters, i.e. $T=50,65,80,95 \mathrm{MeV}$ (cf. ref. [31]), $\langle y\rangle=0,0.3,0.6$. With the given parameters, we determined, with Pluto, the average HADES acceptance for the $\Xi^{-}$hyperon (including the branching ratio of $64 \%$ for the decay of its daughter, $\Lambda \rightarrow \mathrm{p} \pi^{-}$) and its variation within the parameter ranges. Thus, we estimated a systematic error of about $\pm 25 \%$ around the average, purely geometric, $\Xi^{-}$acceptance of $\epsilon_{\mathrm{acc}, \text { sym }}=6.4 \cdot 10^{-2}$ for the above given phase-space parameters. The same Pluto data are processed through GEANT, modeling the detector response. The GEANT data were embedded into real experimental data and then processed through the full analysis chain (using the same topological cuts i) ... viii) as applied to the experimental data). The mean value and the r.m.s. width of the resulting HADES-filtered $\Xi^{-}$rapidity distribution amount to 0.60 and 0.16 , respectively, quite similar to the experimental values. Relating the output to the corresponding input, the total $\Xi^{-}$acceptance $\times$reconstruction efficiency was estimated to $\epsilon_{\text {eff }}=(8.49 \pm 0.24) \cdot 10^{-5}$. As in the $\Lambda$ hyperon analysis [31] we correct for the LVL1 trigger bias w.r.t. minimumbias events, $F_{\mathrm{LVL} 1}=N_{\text {min bias }} / N_{\mathrm{LVL} 1}=1.53 \pm 0.02$, and for empty-track events due to non-target interactions, $F_{\mathrm{MT}}=$ 0.17 . Finally, we note that the experimental $\Lambda$ rapidity distribution [31] does not appear perfectly symmetric. Also, transport model calculations (cf. Fig. 6 of ref. [31]) rather predict a faster yield decrease in the backward hemisphere than is expected from the rapidity-symmetric Pluto distribution. Provided that the $\Xi^{-}$hyperon exhibits a similar asymmetric rapidity distribution as the $\Lambda$, we have to correct the $\Xi^{-}$yield for this difference. We do that by calculating the acceptance ratio of an asymmetric rapidity distribution to the symmetric one. The asymmetric distribution is modelled by a function consisting of two Gaussian distributions. The first Gaussian is the above one describing the $\Lambda$ rapidity density in the region where experimental data points are available and the second Gaussian is a more narrow one $\left(\sigma_{y}=0.13,\langle y\rangle=0.07\right)$ following the transport model predictions at lower rapidities and joining up to the first Gaussian at $y \sim 0.06$. The ratio of the acceptances for asymmetric and symmetric rapidity distributions amounts to $F_{\text {asy }}=\epsilon_{\text {acc, asy }} / \epsilon_{\text {acc, sym }}=1.32 \pm 0.02$. Assuming that the total $\Xi^{-}$acceptance and reconstruction efficiency can be factorized into an acceptance part and and a pure reconstruction-efficiency part which itself does not vary within the, rather limited, acceptance, this factor is used to correct $\epsilon_{\text {eff }}$.

With all the necessary quantities and correction factors at hand, we calculate the $\Xi^{-}$production probability to

$$
\begin{aligned}
P_{\Xi^{-}} & =\frac{N_{\Xi^{-}}}{\left(1-F_{\mathrm{MT}}\right) F_{\mathrm{LVL} 1} N_{\mathrm{LVL} 1} F_{\mathrm{asy}} \epsilon_{\mathrm{eff}}} \\
& =(2.0 \pm 0.4(\text { stat }) \pm 0.3(\text { norm }) \pm 0.6(\text { syst })) \times 10^{-4},
\end{aligned}
$$

where the statistical, absolute normalization, and systematic errors are given.

The dependences of the $\Xi^{-}$yield on the cut values of the various geometrical quantities are displayed in Fig.2. Due to the limited $\Xi^{-}$statistics, only one cut could be varied while all the others are kept fixed to the optimum values (indicated by arrows) yielding the most significant signal. The dependences of experimental data (full circles) and GEANT simulations (open circles) are found to be in good agreement.

Taking the $\Lambda$ production probability of 0.017 per minimumbias event as estimated in ref. [31], the ratio of $\Xi^{-}$and $\Lambda$ production yields can be determined. Such a ratio, when derived from the same data analysis, has the advantage that systematic errors (e.g. the uncertainty of the absolute normalization) cancel to some extent. The ratio amounts to

$$
\frac{P_{\Xi^{-}}}{P_{\Lambda+\Sigma^{0}}}=(1.2 \pm 0.3(\text { stat }) \pm 0.4(\text { syst })) \times 10^{-2} .
$$

Here, the statistical error is dominated by the $20 \%$ error of the $\Xi^{-}$signal, while the systematic error is governed by the stability of the signal against cut variations and by the range of the parameters entering the simulation.

The deduced ratio (2) can be compared with corresponding ratios at higher energies [1,6, 18, 34-36]. Figure 3 shows a compilation of such ratios as a function of $\sqrt{s_{N N}}$. So far, the lowest energy at which a $\Xi^{-} / \Lambda$ ratio is available is $\sqrt{s_{N N}}=2.61 \mathrm{GeV}$, i.e. $630 \mathrm{MeV}$ below the threshold in NN collisions. The corresponding ratio (open circle) was extracted by $\mathrm{HADES}$ from $\mathrm{Ar}+\mathrm{KCl}$ reactions at a beam energy of $1.76 A \mathrm{GeV}$ [12]. A steep decline of the $\Xi^{-} / \Lambda$ production ratio is observed around threshold, where now a second data point (full circle) is available at an excess energy 


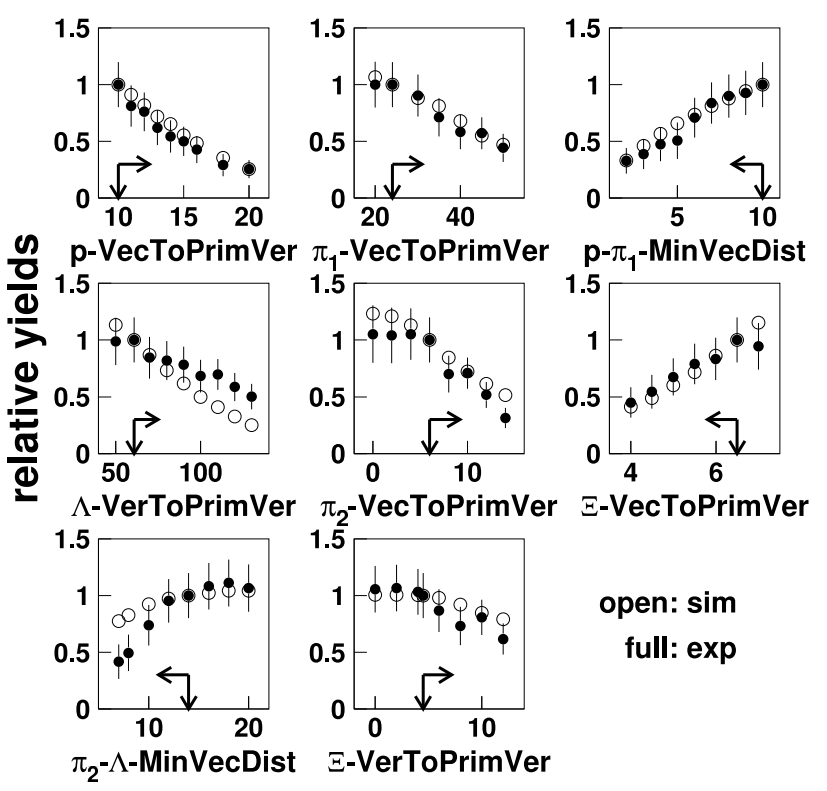

FIG. 2: Relative $\Xi^{-}$yield as a function of the cut value of various $\Lambda$ and $\Xi^{-}$geometrical distances (see text, abscissa units are $\mathrm{mm}$ ). The full (open) circles display the experimental (simulation) data. The vertical and horizontal arrows indicate the chosen cut values and the region of accepted distances, respectively.

of $-70 \mathrm{MeV}$. This allows for comparisons to model calculations (see below). To visualize the energy dependence of the proton-induced data (full curve in Fig. 3), we fitted the corresponding ratios with a function $f(x)=C\left(1-(D / x)^{\mu}\right)^{\nu}$ (with $\left.x=\sqrt{s_{N N}}, C=0.44, D=2.2 \mathrm{GeV}, \mu=0.027, \nu=0.78\right)$, a simple parameterization which may be used to estimate the expected $\Xi^{-} / \Lambda$ ratio in energy regions, where data are not yet available.

The $\Xi^{-} /\left(\Lambda+\Sigma^{0}\right)$ ratio has been investigated within a statistical approach. We performed a calculation with the package THERMUS [37], using the mixed-canonical ensemble, where strangeness is exactly conserved, while all other quantum numbers are conserved only on average by chemical potentials. The optimum input parameters for this calculation (i.e. temperature, $T=(121 \pm 3) \mathrm{MeV}$, baryon chemical potential, $\mu_{B}=(722 \pm 85) \mathrm{MeV}$, charge chemical potential, $\mu_{Q}=(24 \pm 20) \mathrm{MeV}$, fireball radius, $R=(1.05 \pm 0.15) \mathrm{fm}$, and radius of strangeness-conserving canonical volume, $R_{c}=$ $(0.8 \pm 2.1) \mathrm{fm})$ follow from the best fit to the available HADES particle yields $\left(\pi^{-}, \pi^{0}, \eta, \omega, \mathrm{K}^{0}, \Lambda\right)$ in $\mathrm{p}+\mathrm{Nb}$ collisions at $3.5 \mathrm{GeV}$ [29-31]. We obtained a $\Xi^{-}$yield of $1.0 \times 10^{-5}$ and a $\Xi^{-} /\left(\Lambda+\Sigma^{0}\right)$ ratio of $8.1 \times 10^{-4}$ (asterisk in Fig. 3). Both values are significantly lower than the corresponding experimental data.

We also estimated the $\Xi$ production probability within two different transport approaches, both having implemented the aforementioned strangeness-exchange channels. The first ap-

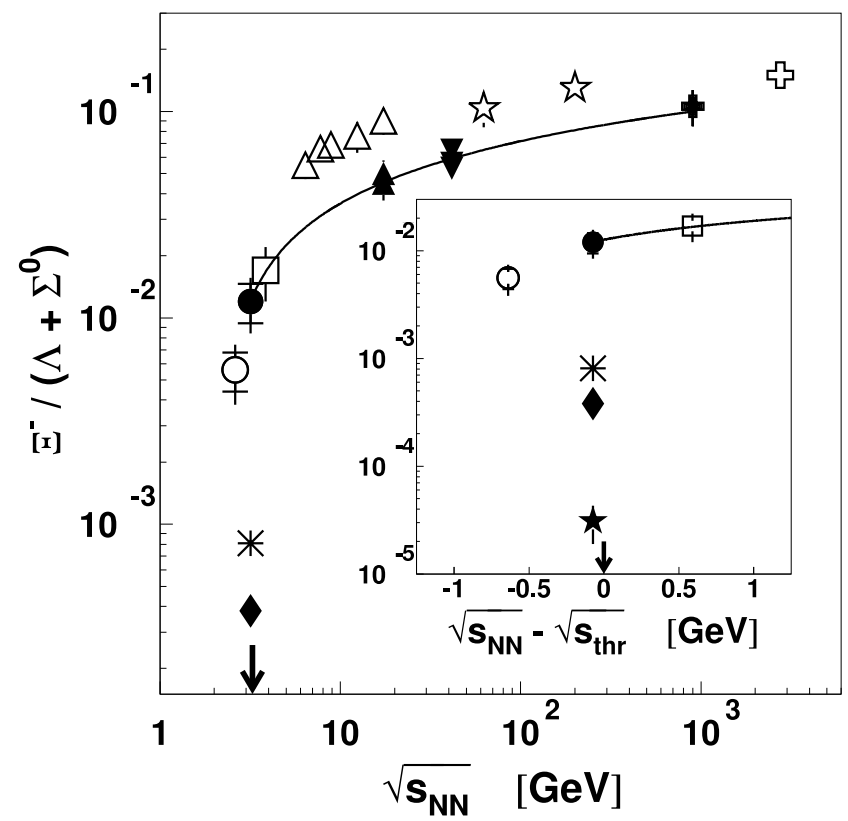

FIG. 3: The yield ratio $\Xi^{-} /\left(\Lambda+\Sigma^{0}\right)$ as a function of $\sqrt{s_{N N}}$ or $\sqrt{s_{N N}}-\sqrt{s_{t h r}}$ (inset). The arrows indicate the threshold in free NN collisions. The open symbols represent data for symmetric heavy-ion collisions measured at LHC [1] 34] (cross), RHIC [2] 3] (stars), SPS [4] 5] (triangles), AGS [6] (square), and SIS18 [12] (circle). The filled cross depicts $p+p$ collisions at LHC [35], while the downward and upward pointing filled triangles are for $\mathrm{p}+A$ reactions at DESY [36] and SPS [18], respectively. The filled circle shows the present ratio (2) for $\mathrm{p}(3.5 \mathrm{GeV})+\mathrm{Nb}$ reactions (statistical error within ticks, systematic error as bar). The full curve is a parameterization (see text) of the proton-induced reaction data. The asterisk, diamond and filled star display the predictions of the statistical-model package THERMUS [37], the GiBUU [38, 39], and the UrQMD [16, 17] transport approaches, respectively.

proach is the UrQMD model [16, 17] (version $\left.{ }^{2} 3.4\right)$. For $\Xi^{-}$ hyperons, we derived a yield of $(6.9 \pm 2.8) \times 10^{-7}$ per event which is more than two orders of magnitude lower than the experimental yield (1) and decreases only by a factor of two, if the channels $\mathrm{YY} \rightarrow \Xi \mathrm{N}$ (with cross sections from [13]) are deactivated; i.e. in the model hyperon-hyperon fusion is of minor importance for $\Xi$ production in proton-nucleus reactions at $3.5 \mathrm{GeV}$. The $\Lambda$ rapidity distribution, however, was fairly well reproduced by UrQMD [31]. The resulting $\Xi^{-} /\left(\Lambda+\Sigma^{0}\right)$ ratio amounts to $(3.1 \pm 1.2) \times 10^{-5}$ (filled star in Fig. 3). The second transport approach we used is the GiBUU model [38, 39] (release ${ }^{3}$ 1.6). We estimated a $\Xi^{-}$ yield of $(6.2 \pm 0.9) \times 10^{-6}$, a value being considerably higher than the prediction by the UrQMD model, but still significantly lower than the experimental yield (1). Also here, the

\footnotetext{
${ }^{2}$ http://urqmd.org

${ }^{3}$ https://gibuu.hepforge.org
} 
total $\Lambda$ yield was quite well (up to $90 \%$ ) reproduced [31]. The $\Xi^{-} /\left(\Lambda+\Sigma^{0}\right)$ ratio amounts to $(3.8 \pm 0.5) \times 10^{-4}$ (filled diamond in Fig. 3. The difference of the results of both transport models may origin from different parameterizations of cross sections of elementary processes.

Summarizing, we investigated the production of the $\Xi^{-}$hyperon in collisions of $\mathrm{p}(3.5 \mathrm{GeV})+\mathrm{Nb}$. For the first time, subthreshold $\Xi$ production is observed in proton-nucleus interactions. Assuming a $\Xi^{-}$phase-space distribution similar to that of $\Lambda$ hyperons, the $\Xi^{-}$yield per event amounts to $(2.0 \pm 0.4$ (stat) \pm 0.3 (norm) \pm 0.6 (syst) $) \times 10^{-4}$. Taking advantage of a recent investigation of $\Lambda$ hyperon production and polarization in the same collisions system [31], the $\Xi^{-} /\left(\Lambda+\Sigma^{0}\right)$ yield ratio of $(1.2 \pm 0.3$ (stat) \pm 0.4 (syst) $) \times$ $10^{-2}$ is derived. Corresponding estimates with the statisticalmodel package THERMUS are significantly lower than the experimental data, by more than an order of magnitude. The GiBUU transport approach predicts a $\Xi^{-}$yield of similar level as that of THERMUS. The UrQMD transport model extremely underestimates the present data, i.e. the $\Xi^{-}$yield is an order of magnitude lower than the GiBUU result. Both transport codes, however, reproduce the pion and single-strange hadron yields fairly well. Hyperon-hyperon scattering processes, YY $\rightarrow \Xi \mathrm{N}$, recently accounted for the temporary puzzle of deep-subthreshold $\Xi$ production in heavy-ion reactions, are found to be of minor importance for subthreshold $\Xi$ generation in proton-nucleus collisions. Hence, a new $\Xi$ puzzle appears, now in proton-nucleus collisions. Probably, direct subthreshold $\Xi$ production happens on the tail of the Fermi distribution of the nucleons within the nucleus, or some of the target protons and neutrons form strongly correlated nucleon pairs, kinematically allowing for either direct $\Xi$ production or the population of an intermediate massive resonance decaying into $\Xi$ baryons. Consequently, more systematic investigations are necessary, including nucleon-nucleon and pioninduced reactions, goals already pursued by HADES.

The HADES collaboration acknowledges the support by BMBF grants 05P09CRFTE, 05P12CRGHE, 06FY171, 06MT238 T5, and 06MT9156 TP5, by HGF VH-NG-330, by DFG EClust 153, by GSI TMKRUE, by the Hessian LOEWE initiative through HIC for FAIR (Germany), by EMMI (GSI), by grant GA CR 13-067595 (Czech Rep.), by grant NN202198639 (Poland), by grant UCY-10.3.11.12 (Cyprus), by CNRS/IN2P3 (France), by INFN (Italy), and by EU contracts RII3-CT-2005-515876 and HP2 227431.

[1] B. Abelev et al. (ALICE collaboration), Phys. Lett. B 728, 216 (2014).

[2] M. M. Aggarwal et al. (STAR collaboration), Phys. Rev. C 83, 024901 (2011).

[3] J. Adams et al. (STAR collaboration), Phys. Rev. Lett. 98, 062301 (2007).

[4] F. Antinori et al. (NA57 collaboration), Phys. Lett. B 595, 68 (2004).

[5] C. Alt et al. (NA49 collaboration), Phys. Rev. C 78, 034918 (2008).
[6] P. Chung et al. (E895 collaboration), Phys. Rev. Lett. 91, 202301 (2003).

[7] Ch. Hartnack, H. Oeschler, and J. Aichelin, Phys. Rev. Lett. 90, 102302 (2003).

[8] C. Fuchs, Prog. Part. Nucl. Phys. 56, 1 (2006).

[9] Ch. Hartnack, H. Oeschler, Y. Leifels, E. L. Bratkovskaya, and J. Aichelin, Phys. Rept. 510, 119 (2012).

[10] L.-W. Chen, C. M. Ko, and Y. Tzeng, Phys. Lett. B 584, 269 (2004).

[11] C. H. Li and C. M. Ko, Nucl. Phys. A 712, 110 (2002).

[12] G. Agakishiev et al. (HADES collaboration), Phys. Rev. Lett. 103, 132301 (2009).

[13] F. Li, L.-W. Chen, C. M. Ko, and S. H. Lee, Phys. Rev. C 85, 064902 (2012).

[14] E. E. Kolomeitsev, B. Tomasik, and D. N. Voskresensky, Phys. Rev. C 86, 054909 (2012).

[15] G. Graef, J. Steinheimer, F. Li, and M. Bleicher, Phys. Rev. C 90, 064909 (2014).

[16] S. A. Bass et al., Prog. Part. Nucl. Phys. 41, 255 (1998).

[17] M. Bleicher et al., J. Phys. G 25, 1859 (1999).

[18] F. Antinori et al. (NA57 collaboration), J. Phys. G: Nucl. Part. Phys. 32, 427 (2006).

[19] V. Koptev et al. (ANKE collaboration), Phys. Rev. Lett. 87, 022301 (2001).

[20] R. Subedi et al., Science 320, 1476 (2008).

[21] K. A. Olive et al. (Particle Data Group), Chin. Phys. C 38, 090001 (2014).

[22] G. Agakichiev et al. (HADES collaboration), Phys. Rev. C 85, 035203 (2012).

[23] G. Agakichiev et al. (HADES collaboration), Phys. Rev. Lett. 98, 052302 (2007).

[24] G. Agakishiev et al. (HADES collaboration), Phys. Rev. C 82, 044907 (2010).

[25] G. Agakishiev et al. (HADES collaboration), Eur. Phys. J. A 47, 21 (2011).

[26] G. Agakishiev et al. (HADES collaboration), Phys. Rev. C 80, 025209 (2009).

[27] G. Agakichiev et al. (HADES collaboration), Eur. Phys. J. A 41, 243 (2009).

[28] G. Agakishiev et al. (HADES collaboration), Phys. Lett. B 715, 304 (2012).

[29] G. Agakishiev et al. (HADES collaboration), Phys. Rev. C 88, 024904 (2013).

[30] G. Agakishiev et al. (HADES collaboration), Phys. Rev. C 90, 054906 (2014).

[31] G. Agakishiev et al. (HADES collaboration), Eur. Phys. J. A 50, 81 (2014).

[32] GEANT 3.21, http://consult.cern.ch/writeup/geant/(1993).

[33] I. Fröhlich et al., Proceedings of Science, PoS(ACAT)076 (2007).

[34] B. Abelev et al. (ALICE collaboration), Phys. Rev. Lett. 111, 222301 (2013).

[35] K. Aamodt et al. (ALICE collaboration), Eur. Phys. J. C 71, 1594 (2011).

[36] M. Agari (HERA-B), PhD thesis, Universität Dortmund, CERN-THESIS-2006-046 (2006).

[37] S. Wheaton, J. Cleymans, and M. Hauer, Comput. Phys. Commun. 180, 84 (2009).

[38] O. Buss, T. Gaitanos, K. Gallmeister, H. van Hees, M. Kaskulov, O. Lalakulich, A. B. Larionov, T. Leitner, J. Weil, and U. Mosel, Phys. Rept. 512, 1 (2012).

[39] J. Weil, H. van Hees, and U. Mosel, Eur. Phys. J. A 48, 111 (2012). 